\title{
METASTABLE STATES AND IRREGULAR BEHAVIOUR IN A JOSEPHSON JUNCTION WITH NONLINEAR RESISTANCE
}

\author{
G. AMBIKA, K BABU JOSEPH, and V. M. NANDAKUMARAN \\ Depanment of Physics, Cochin University of Science \& Technology. \\ Cachin-682 022, India
}

Received 27 December 1990

\begin{abstract}
We consider a resistively shunted Josephson junction with a resistance that depends inversely on voltage. $\mathrm{It}$ is shown that such a junction in the underdamped case can give rise to extremely long-lived metastable states even in the absence of external noisc. We investigate numerically this metastable state and its transition to a chaotic state. The junction voltages corresponding to these states are studied.
\end{abstract}

\section{Introduction}

Nonlinear dissipative systems subjected to external periodic force have been found to exhibit a remarkable variety of modes of response inspite of the apparent simplicity of the equations representing their dynamics. The Josephson junction is an example of such a system, in which the appearance of a specific mode depends sensitively on the parameters characterising the external force. The noisy behaviour in this junction has been extensively studied using numerical methods and analog circuit simulations. ${ }^{1.2}$ The occurrence of irregular behaviour has been attributed to the existence of chaotic solutions to the nonlinear equation of the junction. ${ }^{3.4} \mathrm{~A}$ theoretical study of the tunnel junction is rather complicated due to the nonlinearity of the $I-V$ characteristics. What is usually done is to approximate the behaviour by an equivalent current biased circuit $^{5}$ with a resistance $R$ and a capacitance $C$ in parallel. This resistively shunted junction (RSJ) model, however, is inadequate for describing actual junctions, since $R$ is taken to be a constant. It is true that experimentally measured junction resistance is highly voltage dependent. ${ }^{6}$ A more realistic model would therefore be one in which the resistance depends inversely on voltage, resulting in parabolic $I-V$ curves. In this paper we study this modified RSJ which results in an equation of motion with a quadratic dissipation term. This is found to alter the response of the junction to external periodic force and

PACS Nos.: $74.50+r, 05.45+b, 47.20 . T g$. 
give rise to metastable states with extremely long-lived transients in an underdamped junction. For large values of the driving amplitude, a transition to the chaotic state takes place.

In this context, we would like to mention that such metastable states have been observed in experimental studies of actual junctions. ${ }^{7}$ However, the mechanism for the decay of the current has always been related to the intrinsic thermal fluctuations at finite but low temperatures. The lifetime of the metastable state depends on the resistance $R$ in the low damping regime. In a recent experimental work concerning supercurrent decay in Josephson junctions, Silvestrini et al..$^{8,9}$ consider the effect of dissipation in an underdamped junction. They have established an exponential temperature dependence for $R$. In the present work, we concentrate on the voltage dependence of $R$ and show that metastable states arise mainly due to the nonlinear nature of resistance and can occur even in the absence of noise. The role of noise (or constant bias) is to reduce the barrier height, which in the present case is achieved through the driving force.

In Sec. 2, we briefly summarise the equations of motion representing the junction and discuss the analytic solution that exists in the absence of external force. The details regarding the different modes of response of the system at low frequencies as revealed by studying the phase portraits, Poincare maps and power spectrum are given in Sec. 3. The occurrence of the metastable states is discussed in Sec. 4. Section 5 is devoted to an analysis of the transition to chaos. The results are summarised in Sec. 6.

\section{The Equation of Motion for the Josephson Junction with Nonlinear Resistance}

The electrodynamics of a Josephson junction shunted by a capacitance $C$ and a resistance $R$, and driven by a constant current $I_{\mathrm{dc}}$ and periodic current of frequency $\omega$ is governed by the equation ${ }^{10}$

$$
C \frac{d V}{d t}+\frac{V}{R}+I_{0} \sin \theta=I_{\mathrm{dc}}+I_{1} \sin \omega t
$$

where $\theta$ is the junction phase which is related to the junction voltage $V$ as

$$
\dot{\theta}=2 e V / \hbar
$$

Taking $R$ to be $R_{0} / V$ and using (2), (1) can be simplified to

$$
\ddot{\theta}+k \mid \dot{\theta} j \dot{\theta}+\sin \theta=\rho_{0}+\rho_{1} \sin \omega t
$$


where we use the scaled variables

$$
\begin{aligned}
& t \rightarrow \Omega t \text { with } \Omega=\left(\hbar C / 2 e I_{0}\right)^{1 / 2} ; k=R_{0}\left(\hbar / 2 e C I_{0}\right)^{1 / 2}, \\
& \rho_{0}=I_{\mathrm{d} c} / I_{0} \text { and } p_{1}=I_{1} / I_{0} .
\end{aligned}
$$

Equation (3) in the absence of external driving is known to possess periodic solutions. ${ }^{10}$ With $\rho_{1}=0$ and $y=\dot{\theta},(3)$ can be written as

$$
\frac{d\left(y^{2}\right)}{d \theta}+2 k y^{2}=2 \rho_{0}-2 \sin \theta
$$

which can be solved to give

$$
y^{2}=\rho_{0} / k+4 \cos (\theta+\beta) /\left(1+4 k^{2}\right)^{1 / 2}+C_{1} \exp (-2 k \theta)
$$

with $\tan \beta=2 k$. The steady state solutions after transients have died out can be integrated to obtain $\theta$ in terms of Jacobian elliptic functions.

When $\rho_{1} \neq 0$, the system shows a rich variety of interesting dynamics. The method of Melnikov analysis has been used to predict the lowest threshold for chaos in this system. " We carry out detailed investigations regarding the possible routes to chaos, the nature of the chaotic state, etc. in this model.

\section{Profiles of Motion and Response Modes}

The dynamics of the junction described by (3) can be simulated by a particle moving dissipatively in a potential $U(\theta)$ given by

$$
U(\theta)=1-\left(\cos \theta+p_{1} \theta \sin \omega t+p_{0} \theta\right) .
$$

This corresponds to ' $a$ ' series of potential wells whose barrier heights are modified by the biasing parameters $\rho_{1}$ and $\rho_{0}$. The pitch of this screw type motion increases with $\rho_{1}$ until for a critical value $\rho_{c}$ the trajectory escapes from the central well. The junction voltage in this frequency region shows a square wave pattern, which under finer scaling reveals periodic motions in two widely differing time scales (Fig. 1b).

\section{Metastable State}

In the frequency range $0.2<\omega<1$, we observe that the system exhibits a metastable oscillatory mode characterised by a trajectory that continues indefinitely for a long interval of time (Fig. 2a). The continuous disturbance introduced by the quadratic damping prevents the system from forming stable limit cycles. The small fluctuations associated with the transient nature of the motion leads to broading of peaks in the power spectrum of voltage. This is clear from Fig. $2 b$ where the Fast Fourier Transform (FFT) ${ }^{12}$ of the junction voltage is given. The 


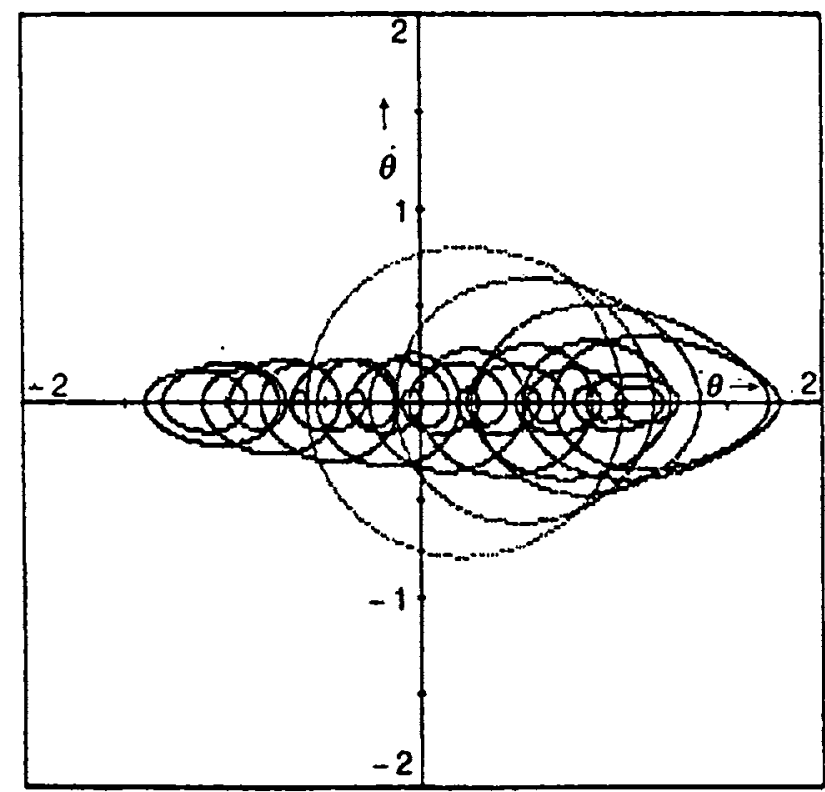

Fig. 1a. The screw type trajectory plotted in the $\theta-\dot{\theta}$ plane for $\omega=0.05$ with $k=0.1, \rho_{0}=0$ and $\rho_{1}=0.9$.

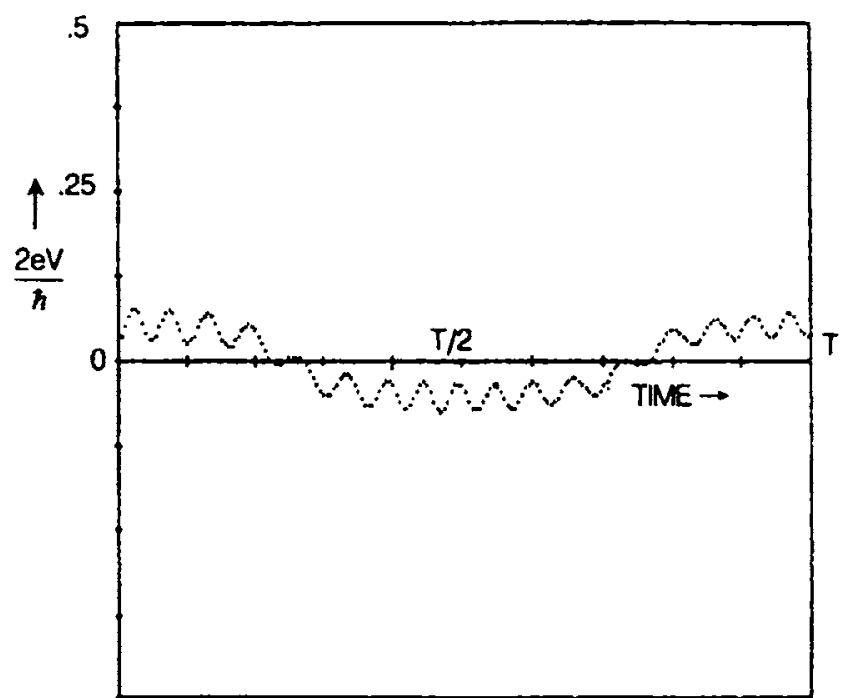

Fig. Ib. The variations in the junction voltage $V$ for the parameters in Fig. Ia. The voltage multiplied by $(2 e / h)$ is plotted along the $Y$ axis white time is along the $X$ axis. $T$ is the period of the extermal force $(-2 \pi / \omega)$. 
corresponding Poincare map shows a very slowly advancing curve. A quantitative characterisation of this metastable state, we feel, is possible through the Lyapunov Characteristic Exponent (LCE), which measures the average rate of convergence or divergence of nearby trajectories. Using the conventional techniques, ${ }^{13}$ we compute the maximum LCE $\lambda_{\max }$ and find that for the metastable state the value of $\lambda_{\max }$ is very small, $\approx 10^{-2}$. Whether it is central well has $-\pi / 2<\theta<\pi / 2$ with the minimum at $\theta=0$. The motion of the system in general is of the oscillatory type confined to the central well for low values of $\rho_{1}$, while for large values of $\rho_{1}$ the system escapes from the well and describes running modes over the potential maxima. We find that the response of the system, both before and after the escape, depends primarily on the frequency of the driving current $\omega$. Depending on the value of $k$, after escaping from the well, the system may get itself trapped in any of the successive minima too. Chaotic states arise due to random shuttling between running and oscillatory modes.

For our analysis, (3) is rewritten as a system of three first order equations. With $\theta=x_{1}$ we have

$$
\begin{aligned}
& \dot{x}_{1}=x_{2}, \\
& \dot{x}_{2}=-k\left|x_{2}\right| x_{2}-\sin x_{1}+\rho_{0}+\rho_{1} \sin x_{3}, \\
& \dot{x}_{3}=\omega .
\end{aligned}
$$

This constitutes a three dimensional dissipative system with quadratic damping. We restrict the analysis to the case of extreme underdamping with $k=0.1$. For low values of $\omega$, namely, $0.02<\omega<0.2$, the system behaves adiabatically and the profile of motion has structure on a very fine scale. The trajectory shows a screw type motion around the $\theta$ axis (Fig. la) which is being repeated as an oscillation over a certain region with the periodicity of the applied force. The amplitude of the motion decreases after each oscillation but the system refuses to settle down to a stable trajectory. The positive or negative depends, in this context, on $\omega$. Metastable states can thus decay into a stable trajectory or develop into a chaotic attractor, after a sufficiently long interval of time. If $\lambda_{\max }$ is positive, the ultimate state is chaotic while a negative $\lambda_{\max }$ takes the trajectory asymptotically to stable limit cycle. Here the rate of convergence or divergence is seen to follow a power law rather than an exponential behaviour that is usually observed near limit cycles or chaotic attractors.

The transient nature of the solution to (3) is clear from the last term in (6). What the external periodic force does is to prevent the transients from dying down fast and thus sustain them, thereby resulting in metastability. This is especially so for low damping which we consider here. The fluctuations in the 


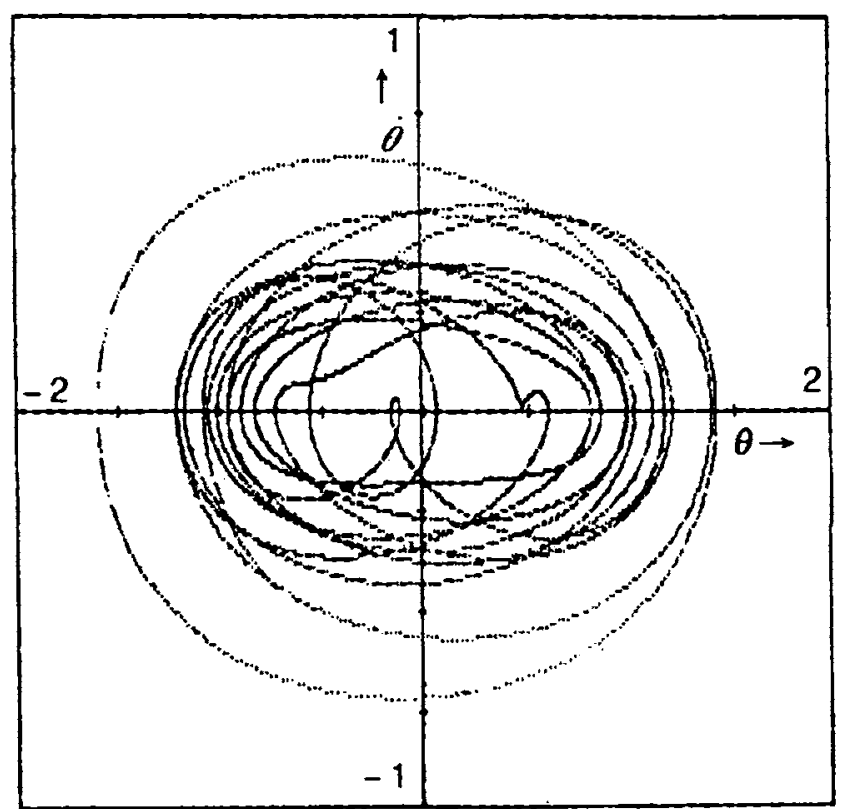

Fig. 2a. Metastable trajectory observed for $\omega=0.6, \rho_{0}=0, k=0.1$ and $\rho_{1}=0.3$.

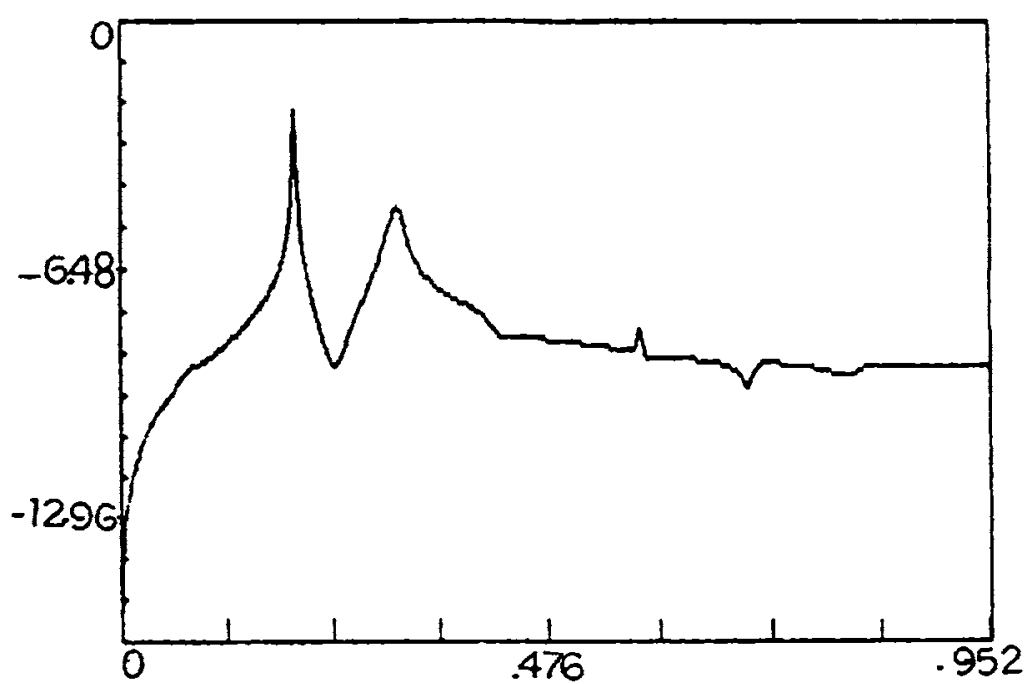

Fig. 2b. The power spectnm of the metastable state using FFT. Log(power) is plotted along the $Y$ axis with frequency along the $X$ axis. 


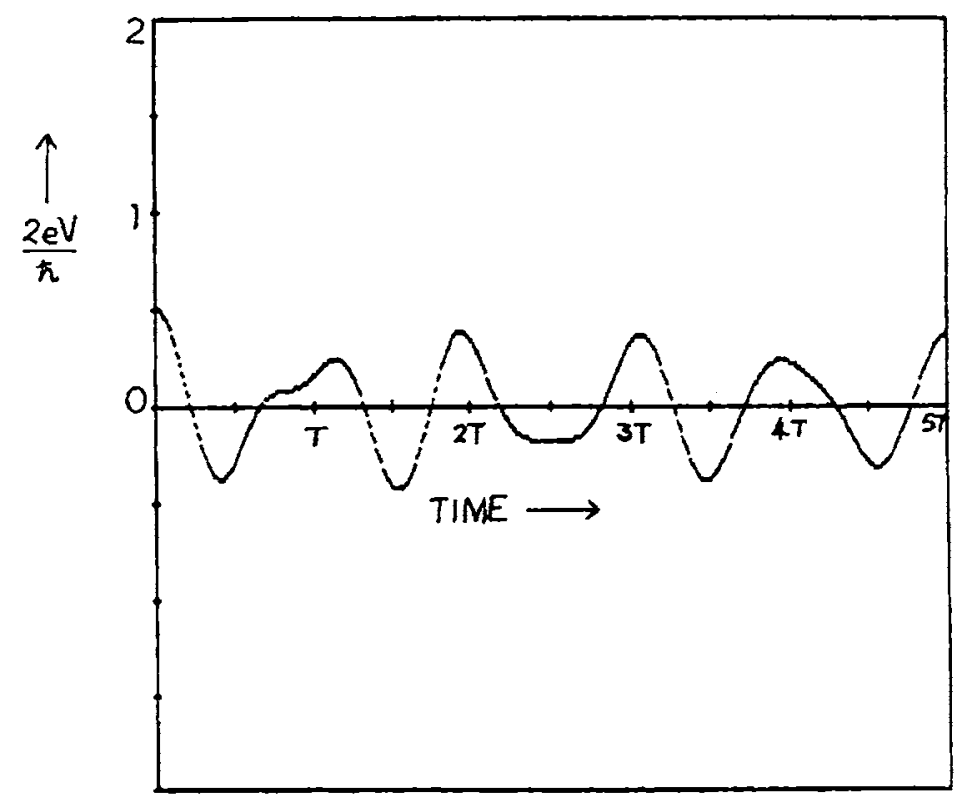

Fig. 2c. The fuctuations in the junction voltage in the metastable state corresponding to the parameters in Fig. 2a.

junction voltage corresponding to this state is given in Fig. 2c. As $\omega$ is increased further; the metastable nature of the trajectory prevails, but $\lambda_{\max }$ becomes positive, though small.

\section{Transition to Chros}

In all the frequency ranges investigated by us a transition to a chaotic state with positive $\lambda_{\max }(0.4-0.5)$ takes place after escape from the central well. This happens at a critical value of $\rho_{1}$, viz. $\rho_{c}$, which depends on $\omega$ and $p_{0}$. For low values of $\omega$, after escape from the well, the trajectory suns over several hundreds of potential wells and, in accordance with the terminology introduced by Yamaguchi, ${ }^{14}$ we therefore call this state running chaos. The fluctuations in the voltage for this state is clear from Fig. 3a. The chaotic nature is due to the fact that the point where the trajectory turns back to the central region is random. This results in a random shuttling between the positive and negative running modes through damped oscillations. As $\omega$ is increased, the nature of the chaotic state remains the same. However, now the trajectory runs only over a few potential wells and so is called oscillatory chaos. The junction voltage variations are given in Fig. 3b. The trajectories and their FFTs corresponding to both these types of chaos are seen in Fig. 4. 


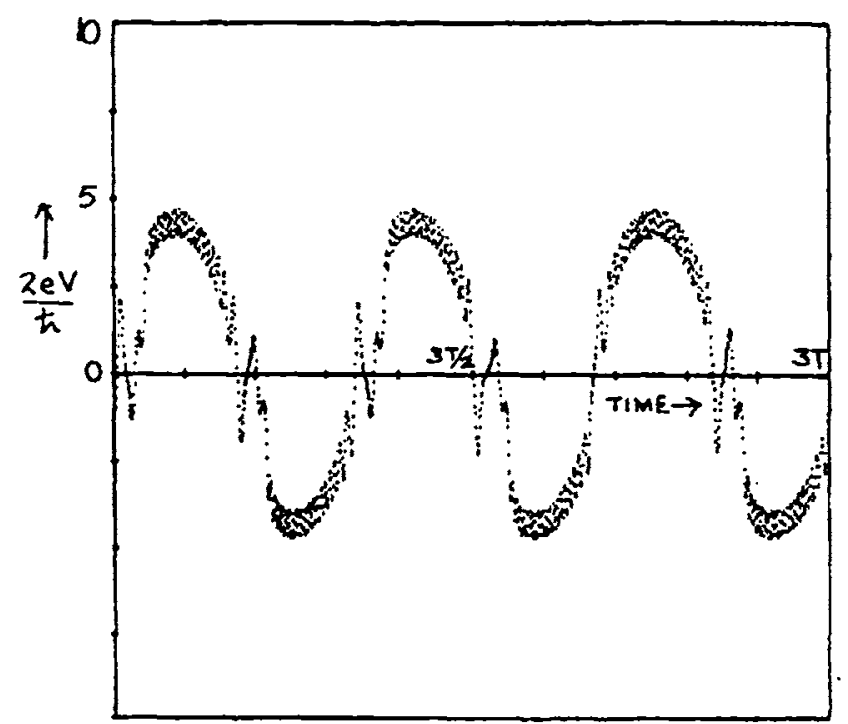

Fig. 3a. The junction vollage in the chaotic stale after escape from the central well. Here $\omega=0.05$, $k=0.1, \rho_{0}=0$ and $\rho_{1}=1.2$.

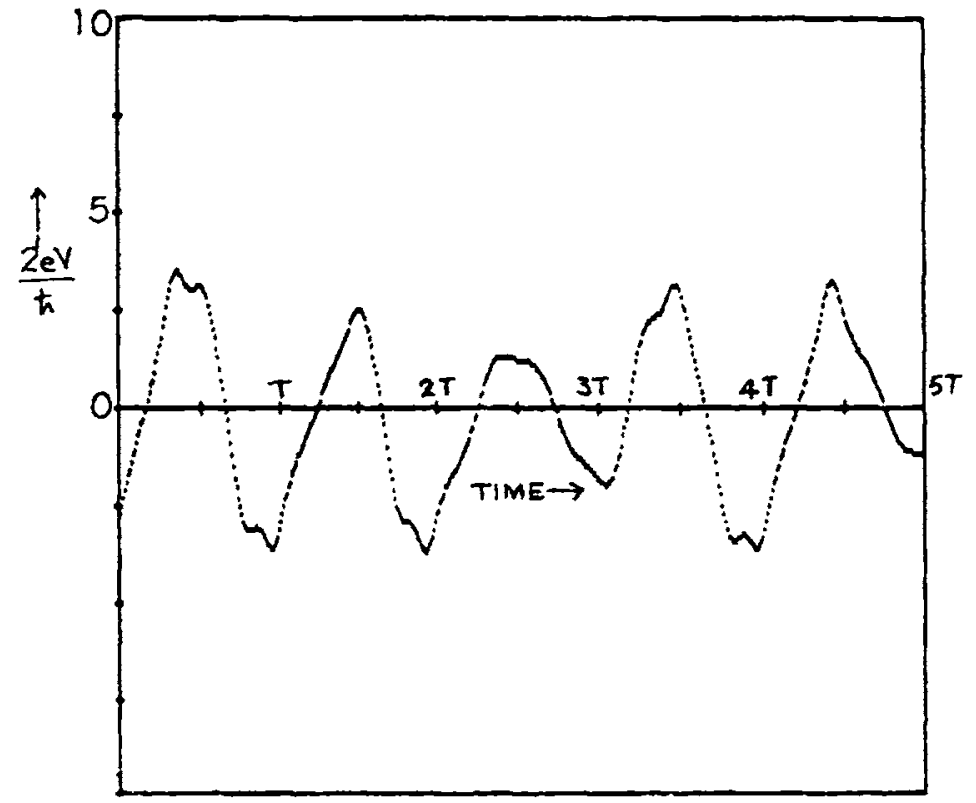

Fig. 3b. The voltage corresponding to a frequency $\omega=0.6$, where oscillatory chaos is observed $k=0.1, \rho_{0}=0, \rho_{1}=2$. 


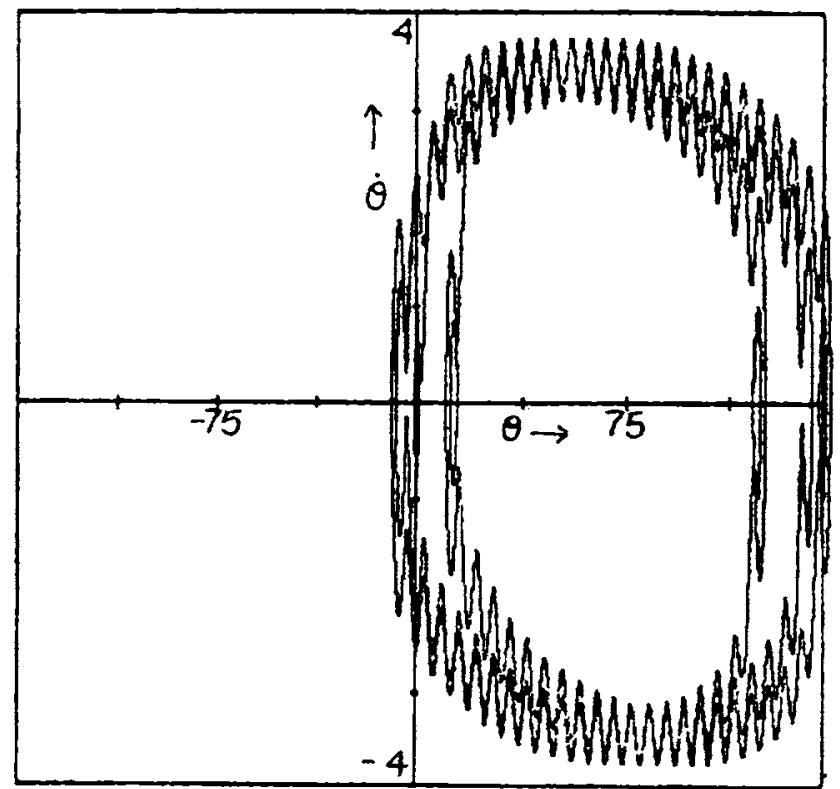

Fig. 4a. The running chaos observed in the low frequency regime with $\rho_{1}=1.2$. Note that the value of 0 goes upto 150 .

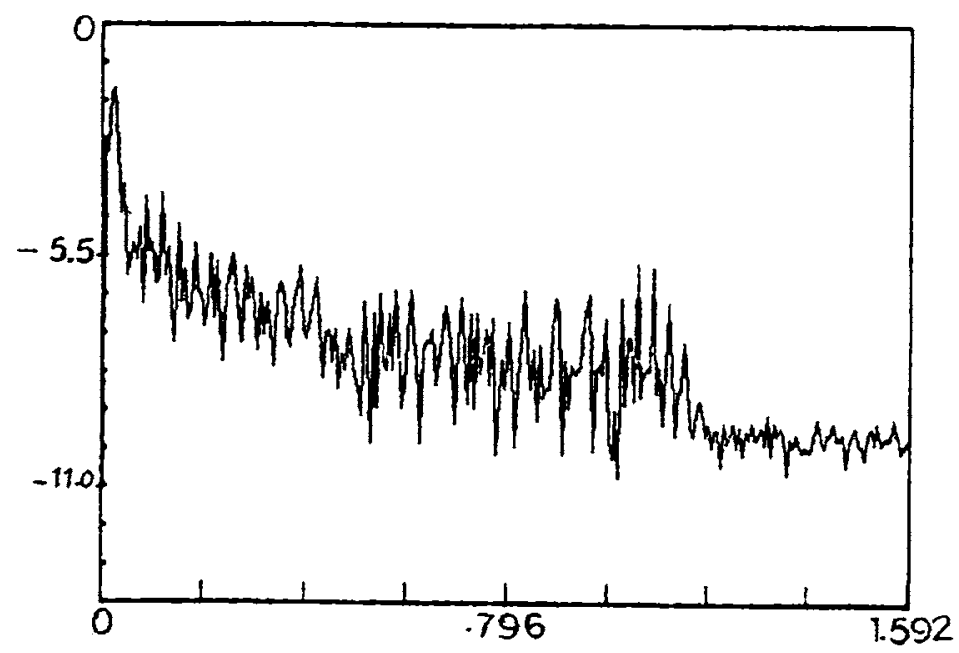

Fig. 4b. The FFt corresponding to running chaos. 


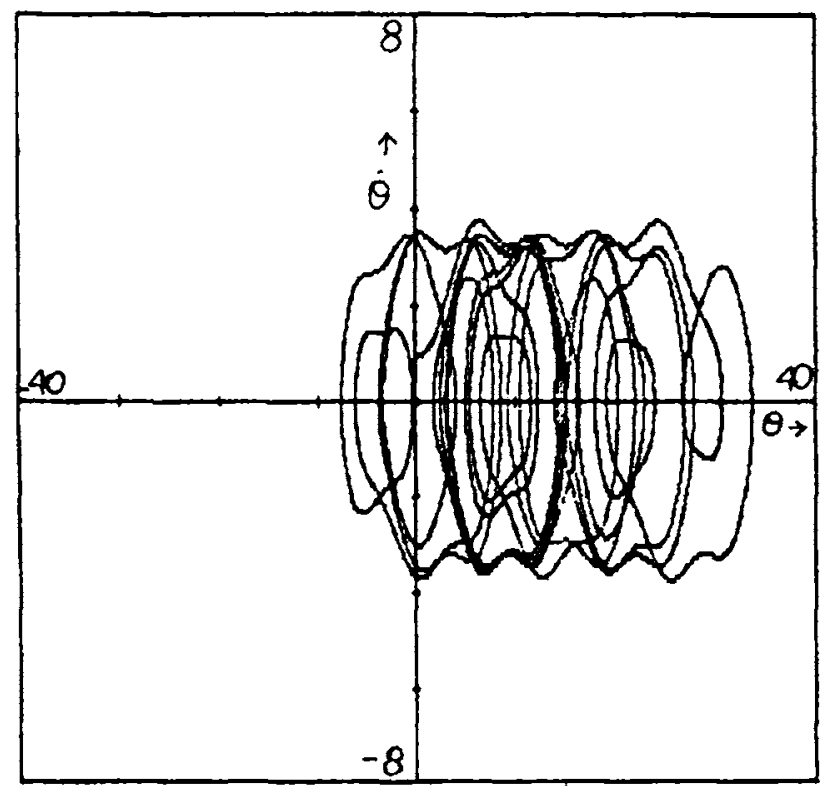

Fig. 4c. The oscillatory type chaos at $\omega=0.6$ and $\rho_{1}-2$.

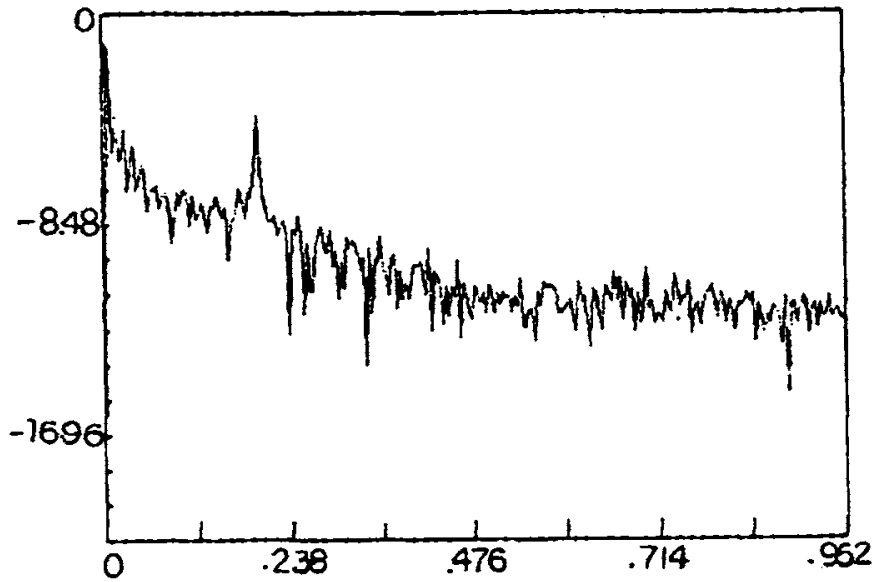

Fig. 4 d. The FFT of the oscillatory chaotic state.

\section{Conclusion}

We have investigated numerically a modified RSJ model for a driven Josephson junction with low damping. The mechanism of the onset of chaos in this system is similar to that in the usual RSJ model. But in the latter model, stable periodic limit cycles exist before chaos and they do undergo the period 
doubling route. ${ }^{3}$ However, in the system considered here, stable limit cycles exist only in a very narrow frequency range, from 4 to 5.5 for large values of $k \approx 2$. Moreover, the running modes are unstable even before chaos and the oscillatory modes inside the central well are not stable but metastable. In our computations we have kept $\rho_{0}=0$. But for nonzero $\rho_{0}$ qualitatively the same behaviour is observed with the escape from the well taking place at a lower value of $\rho_{1}$. The role of noise is also to shift the value of $\rho_{c}$. The metastable nature arises mainly due to the nonlinear nature of the resistance. Both the running and the oscillatory types of chaos are found to occur in this system.

\section{Acknowledgements}

One of us (G.A.) would like to acknowledge the financial support of the Council of Scientific and Industrial Research, New Delhi through the award of a research associateship.

\section{References}

1. B. A. Huberman and J. P. Crutchfield, Phys. Rev. Lett. 43 (1979) 1743.

2. R. L. Kautz and R. Monaco, J. Appl. Phys. 57 (1985) 875.

3. D. D' Humieres, M. R. Beasley, B. A. Huberman, and A. Libchaber, Phys. Rev. A26 (1982) 3483.

4. B. A. Huberman, I. P. Crutchfeld, and N. H. Packard, Appl. Phys. Lett. 37 (1980) 750.

5. W. C. Stewart, Appl. Phys. Lett. 12 (1968) 277.

6. L Solymar, Superconductive Tunnelling and Applications, (Chapman and Hall Ltd., London, 1972).

7. A. I. Larkin and Y. N. Orchinmikov, Phys. Rev. B28 (1983) 6281.

8. R. Christiano and P. Silvestrini, Phys. Lett. A133 (1988) 347.

9. P. Silvestrini, O. Liengme, and K. E. Gray, Phys. Rev. B37 (1988) 152S.

10. N. F. Pedersen and K. Saermark, Physica 69 (1973) 572.

11. M. Bartuccelli, P. L Christiansen, N. F. Pedersen, and M. P. Soerensen, Phys. Rev. B33 (1986) 4686.

12. E O. Brigham, The Fast Fourier Transform, (Prentice Hall Inc. Englewood, Cliffs 1974).

13. W. H. Steeb and J. A. Louw, Chaos and Quantum chaos, (World Scientific, 1986), p. 42.

14. Y. Yamaguchi, Prog. Theor. Phys. B28 (1983) 6281. 